\title{
Implementing a new drug record system: a qualitative study of difficulties perceived by physicians and nurses
}

\section{S E Andersen}

Correspondence to:

S E Andersen MD,

Rigshospitalet, Department of Clinical Pharmacology Q7642, Blegdamsvej 9, DK-2100 Copenhagen $\varnothing$, Denmark; sean@rh.dk

Accepted 6 August 2001

\begin{abstract}
Objectives: To identify organisational difficulties faced by physicians and nurses when using drug prescribing sheets for recording both drug prescriptions and drug administration.

Design: Qualitative interview study.

Setting: Two general internal medicine wards.

Participants: Seven physicians and eight nurses.

Main outcome measures: Difficulties explicitly identified by the participants during the interviews. Results: The implementation of procedures conflicted with existing structure, culture, and routines. Insufficient competence within the system to use the drug prescribing sheets created resistance and made people down the line create their own interpretations and solutions to the problems they faced. A total of nine problems were identified: (1) insufficient knowledge and uncertainty about procedures, (2) ignorance of sources of error, (3) unclear responsibilities, (4) low community spirit, (5) insufficient communication, (6) clinician autonomy and low acceptance of change, (7) strong professional identity, (8) low priority task, and (9) logistical problems.

Conclusions: Unawareness of procedures, insufficient dissemination of knowledge, and insufficient cooperation and scepticism among those who put drug handling into practice is likely to have an impact on the quality of health care. The identification of these obstacles may help managers to improve the quality of the drug handling process on internal medicine wards and make it possible to select a framework for changing the clinical behaviour of doctors and nurses.
\end{abstract}

D anish clinicians traditionally dictate drug orders or write drug prescriptions in rough. Nurses then copy information about drugs either from rough drafts or from progress notes, typed and organised by secretaries, to nursing records or medicine lists which are used for drug administration. As copying is difficult, medicine lists are likely to differ from medical records. ${ }^{1}$ In agreement with previous studies, ${ }^{2-4}$ we have shown that errors in copying affects more than half of inpatients. ${ }^{5}$ Erroneous copying and other defects in systems to make drug and patient information accessible at the time it is needed may cause adverse drug events. ${ }^{6}$

Changing the organisation ${ }^{7}$ and the systems by which drugs are ordered and administered may reduce the number of drug related accidents. ${ }^{6-10}$ To reduce the complexity of the drug prescribing process in hospitals ${ }^{11-14}$ and facilitate data retrieval, ${ }^{15}$ new drug prescribing sheets have been designed. Drug prescribing sheets are single documents which are used by physicians to write medication orders by hand, and by nursing staff to determine the dose and to record their administration. They are preprinted with spaces for the drug name, dose etc, and for each administration to be signed for, and can be used without transcription. Drug prescribing sheets have been used in some countries for more than 30 years. Studies have shown that the implementation of drug prescribing sheets in the clinical setting may improve the quality of health care, mainly by reducing the number of errors in drug administration. ${ }^{14} 16-18$

Achieving quality improvement by the adoption of drug prescribing sheets relies on effective implementation of the new system. Studies on the implementation of guidelines have indicated that no single implementation strategy is superior to achieve changes in behaviour. ${ }^{19}$ There are numerous approaches, all of which may be more or less effective depending on the changes sought, the target group, and the barriers found. The adoption of drug prescribing sheets forces physicians and nurses to develop interdisciplinary collabora- tion by modifying established routines and workflow patterns. However, changing drug prescribing procedures is likely to create dissatisfaction among staff since freedom to order medications and freedom to set volume of work and scheduled priorities is related to perceived clinical freedom and physician satisfaction..$^{20}$ Identification of the difficulties and obstacles in changing drug prescribing procedures is crucial for its successful implementation because it may help managers to become aware of professional concerns ${ }^{22}$ and select an ideal framework for improving the clinical behaviour of staff. ${ }^{23} 24$

This qualitative study aimed to identify the difficulties faced by physicians and nurses when using drug prescribing sheets.

\section{METHODS \\ Setting}

The study was conducted on two general internal medicine wards of 56 and 74 beds located in general hospitals serving populations of 150000 and 130000 , respectively. On l October 1998 and 1 June 1999 two different drug prescribing sheets were taken into general use on the study wards. The drug prescribing sheets differed in size, but both were slightly modified versions of the same master char $\mathrm{t}^{14}$ and were used according to similar principles. Representatives of the medical and nursing staff were included in the entire development and implementation process. The wards had similar ward based medication supply systems: all drugs frequently used were stocked in the wards but some drugs for infusion or injection were dispensed for individual patients. Nurses made drug administration rounds 4-6 times daily. In neither the old nor the new system were pharmacy technicians or clinical pharmacists attached to the wards.

\section{Participants}

During February and March 2000 in depth interviews with seven physicians and eight nurses were conducted in the two 
Box 1 Interview schedule

Introduction

- How do you use the drug prescribing sheet in your daily work?

Technology

- What is your general view of the drug prescribing sheet?

Tasks

- Has the implementation of the drug prescribing sheet had any impact on your daily tasks?

- What differentiates completing drug prescribing sheets from what you did previously?

Structure

- Which working procedures are related to the drug prescribing sheet?

- How does the interdisciplinary collaboration on drug prescribing work?

- How is the interdisciplinary collaboration coordinated?

- Who is responsible for the drug prescribing sheet in practice?

Personnel

- How much time do you spend daily on drug prescribing or drug administration?

- Has this changed?

- What is your role in drug prescribing or administration?

- In your opinion, is the time spent on drug prescribing or drug administration worth while?

- Do you have any idea of how your skills as a health professional can be valued?

- How do you imagine the task of prescribing or administering drugs would be undertaken under optimum conditions?

hospitals 8 and 16 months, respectively, after the introduction of drug prescribing sheets. A chain sample approach ${ }^{25}$ was used for locating key informants who had gained experience with the drug prescribing sheets. Demographic characteristics and occupation guided the sampling. The aim of the sampling process was to include participants with a wide range of practical experience. Thus, managing medical or nursing staff who had little or no practical experience with the new system were not eligible. Anonymity of the interviewees was guaranteed.

\section{Interviews}

Semi-structured interviews were conducted with informants at different levels of seniority. The interview schedule set out in box 1 was developed following discussions with interdisciplinary staff and two pilot interviews and consisted of questions addressing all four elements of Leavitt's system model $^{26}$ : technology, structure, tasks, and personnel. Inquiries were made flexibly to allow each participant to contribute according to their role and experience. Where required, confirmatory questions were interposed to enhance communicative validity. ${ }^{27}$ No further interviews were conducted when no new themes occurred. Each interview lasted 25-85 minutes and was entered as a sound file directly into a laptop computer during the interview. After each session a written summary of the author's interpretation was sent to the informants asking them to comment on the findings.

\section{Data analysis}

Data were analysed according to Giorgi's phenomenological approach with Malterud's modification ${ }^{28} 29$ in four stages: (1) obtaining an overall impression by repeated listening to sound files and review of written summaries; (2) identifying units of meaning representing different aspects of the interviewees' experiences and coding for these; (3) condensing and abstracting the meaning within each of the coded groups; and (4) summarising the content of each coded group to gain descriptions. The code-and-retrieve software ${ }^{30}$ package кIT version 2.0 (CVS Information System) was used, allowing codes to be attached directly to the sound files. Coded groups were subsequently transcribed. The findings were repeatedly compared with the original sound files and written summaries. When possible the information was validated by comparing the perspectives of physicians and nurses. ${ }^{31}$ For comparison, information on the introduction and training policies was subsequently collected from the wards studied. Moreover, information was collected from working parties on the drug prescribing sheet and compared during the analysis process. To verify alternative or erroneous use a sample of drug prescribing sheets was reviewed.

Data from this study will be provided to local hospital managers, ward managers and senior lecturers in clinical pharmacology to support decisions about implementing drug prescribing sheets.

\section{Approval}

The study was approved by the local ward managers and the Data Surveillance Authority and conducted according to local ethical regulations.

\section{RESULTS}

All staff who agreed to participate in the study gave consent except for one staff specialist. The interviewees are shown in table 1 and the hierarchical system of Danish medical staff is given in the legend.

The interviewees described various issues but the dominant theme was drug prescription procedures. The analysis focused only on difficulties relating to the drug prescribing sheet which was explicitly identified by the participants during the interviews. A total of nine difficulties were identified (box 2).

\section{Lack of knowledge and competence Learning by doing}

Uncertainty about how to use the drug prescribing sheet was an important difficulty. Several nurses reported that the physicians filled in prescriptions inconsistently and erroneously. Four physicians supported this opinion. The physicians had been trained differently: two had not been trained, two had been offered a training programme, and the remainder had briefly been introduced to the new procedures. All had felt prompted to take steps on own initiative to improve practice. One physician said:

"I have made some home made solutions to the drug sheet problems I have faced. At any rate, I find them reasonable" (Senior house officer (5))

Examples of home made solutions included various ways of recording drug doses and schedules, reducing doses, stopping treatments, and entering reasons for prescribed treatments in the medical records. Two nurses expressed the opinion that insufficient knowledge of pharmacology and drug recommendations by some of the physicians contributed to errors. The nurses preferred mutual training and were surprised that physicians did not train each other. Several nurses had been involved in the physicians' training and felt it was paradoxical because they saw drug prescription solely as the task of physicians. They suggested that a training programme should be included in the introduction of physicians to the ward.

\section{Ignorance of sources of error}

The participants felt that some of the physicians seemed to ignore sources of drug error and they gave examples of erroneous procedures that could possibly hamper drug safety-for example, some physicians recorded prescriptions in the margin of completed sheets instead of starting on a new sheet. To minimise the workload one physician had suggested cutting out sections of completed sheets and pasting them onto new sheets with adhesive tape. He said that the ward managers were considering his suggestion. Two physicians 
Table 1 Characteristics of interviewees

\begin{tabular}{|c|c|c|c|c|c|}
\hline & Sex & $\begin{array}{l}\text { Age } \\
\text { (years) }\end{array}$ & $\begin{array}{l}\text { Years since } \\
\text { graduation }\end{array}$ & $\begin{array}{l}\text { Duration of present } \\
\text { appointment }\end{array}$ & $\begin{array}{l}\text { Experience of using drug } \\
\text { prescription sheet (months) }\end{array}$ \\
\hline \multicolumn{6}{|l|}{ Ward 1} \\
\hline Nurse (1) & $\mathrm{F}$ & 25 & 1.5 years & 1 year & 8 \\
\hline Nurse (2) & $\mathrm{F}$ & 26 & 2.5 years & 1.5 years & 8 \\
\hline Nurse (3) & $\mathrm{F}$ & 28 & 3 years & 3 years & 8 \\
\hline Nurse (6) & $\mathrm{F}$ & 47 & 25 years & 8 years & 8 \\
\hline Nurse (8) & M & 26 & 2 years & 1.5 years & 9 \\
\hline Specialist registrar (1) & $\mathrm{F}$ & 42 & 6 years & 1 year & 8 \\
\hline Staff specialist (2) & M & 42 & 14 years & 3 years & 8 \\
\hline House officer (6) & $\mathrm{F}$ & 28 & 9 months & 5 months & 5 \\
\hline Staff specialist (7) & $\mathrm{F}$ & 42 & 16 years & 11 years & 9 \\
\hline \multicolumn{6}{|l|}{ Ward 2} \\
\hline Nurse (4) & $\mathrm{F}$ & 33 & 3 years & 3.5 years & 22 \\
\hline Nurse (5) & $\mathrm{F}$ & 38 & 11 years & 5 years & 22 \\
\hline Charge nurse (7) & $\mathrm{F}$ & 45 & 23 years & 13 years & 17 \\
\hline Senior house officer (3) & M & 31 & 2.5 years & 5 months & 5 \\
\hline Staff specialist (4) & M & 44 & 17 years & 9 months & 9 \\
\hline Senior house officer (5) & M & 33 & 6 years & 8 months & 8 \\
\hline
\end{tabular}

had resumed the habit of recording drug orders twice. When asked to explain, one gave safety reasons while the other said:

"Writing the same thing in two places presumably satisfies the requirements." (Staff specialist (4))

In the new system secretaries do not type out prescriptions. Both physicians and nurses reported that illegible prescriptions had become a widespread problem. The physicians explained illegibility and inconsistency by poor hand writing skills and pressure of work.

\section{Conflicts with existing organisational structure and culture \\ Unclear responsibilities}

Discussions between physicians and nurses occurred frequently concerning uncertainty as to the responsibility for completing the drug prescribing sheets. Nurses from both wards described this as a significant problem. Although some of the participants regarded collaboration as frictionless, both physicians and nurses felt demotivated by these discussions. One nurse said:

"If we could collaborate, I think the technical problems would be of no particular importance." (Nurse (2))

The nurses expressed the view that they were particularly inconvenienced by erroneous prescribing because they were dependent on complete drug lists to administer drugs safely. They felt prompted to check up on the physicians and almost daily each nurse had to call a physician to resolve drug problems, causing delays or postponement of nurses' tasks. These

\section{Box 2 Problems identified by nurses and physicians}

Lack of knowledge and competence

- Insufficient knowledge and uncertainty about procedures

- Ignorance of sources of error

Conflicts with existing organisational structure and culture

- Unclear responsibilities

- Low solidarity

- Insufficient communication

- Clinician autonomy and low acceptance of change

- Strong professional identity

Conflicts with existing routines

- Low priority task

- Logistical problems checks usually led to a correction of the errors pointed out but produced no general impact on the prescribing behaviour of physicians.

\section{Low community spirit}

A low level of community spirit among physicians was an obstacle to standardisation of prescribing procedures. The physicians generally worked individually; they were cautious in discussing the quality of care given by colleagues and preferred to correct errors without telling their colleagues. They withheld justified critique if a colleague seemed overburdened. A junior physician expressed the opinion that physicians were more concerned about personal careers than issues of cooperation. In one of the wards rivalry and competition within the physician group was pronounced:

"Community spirit is high only when we are opposing other staff groups, otherwise we are rivals." (Staff specialist (7))

A staff specialist said that a feeling of community spirit presupposed years of cooperation. Although senior physicians communicated with junior doctors, awareness of the hierarchical structure had an important effect on the junior doctors and they usually restricted their contact with senior team members:

"If I'm going to ask a superior physician the question has to be very relevant. It is relevant, of course, that prescriptions are illegible, but it is somehow on another level." (Senior house officer (5))

Unlike physicians, nurses reported a high degree of community spirit and considerable uniformity in their attitude towards the prescribing sheets.

\section{Insufficient communication}

The participants felt that insufficient communication impeded the sharing of experiences and prevented harmonisation of the new procedures. The ward conference was considered the formal forum for debate of administrative and patient related subjects. However, both physicians and nurses preferred trading opinions about the drug prescribing sheets informally. Three physicians remembered that problems about drug sheets had been mentioned on the ward conference but the discussion had been brushed aside by "wisecracks". There was an implicit threshold determined by ideas of importance which was more evident if topics were discussed on a regular basis:

"The general idea is that we are above moaning about the drug sheets." (Senior house officer (3)) 
Only if the topic of discussion was of very high significance were written messages on conference decisions distributed to non-participating colleagues.

\section{Clinician autonomy and low acceptance of change} Several participants reported a high degree of clinician autonomy and a low acceptance of change. They expected to be included in decision making processes and perceived that they had not been involved in the adoption of the drug prescribing sheet. Two physicians felt that physicians were generally not involved because the permanent staff of nurses, secretaries, and hospital porters was having a greater say. Some of the participants expressed the view that the implementation process was of low management priority because the modification proposals were generally not accepted. Several felt increasingly overburdened by the workload created by their managers for administrative reasons and saw the drug prescribing sheet as an example of bureaucratisation. One registrar said:

"Therapy is snowed under with administration. It is a clothes-peg factory." (Specialist registrar (1))

The participants considered whether illegibility was an expression of dissatisfaction with changes:

"I haven't a clue, but one would think that people write illegibly because they are annoyed with the sheets" (Staff specialist (2))

Three physicians had noticed that newly hired colleagues seemed to have a high degree of acceptance of new procedures, and they predicted that resistance would subside gradually as a result of staff turnover. One male nurse disagreed and argued that, by providing insufficient training, the permanent staff signal to new members that drug prescribing sheets are of low priority.

\section{Strong professional identity}

Both nurses and physicians had distinct perceptions of their professional roles and the tasks they were expected to undertake. They all expressed clear positions on division of labour between nurses and physicians. Some of the nurses did not regard drug dispensing and administration as their task by right, but within the nurse group there was inconsistency on this topic. Documenting drug administration was the nurses' task, but some of the physicians felt that the task of completing nurses' drug lists had been shifted to physicians. They said that writing drug orders by hand was not a task under their subject area. One registrar regarded it a paradox that physicians were expected to complete $x$ ray request charts but were not expected to fill in drug orders. A staff specialist felt that, rather than making physicians fill in prescriptions by hand, nurses should be taught to copy accurately. Others expressed the opinion that either secretaries or nurses should record drug prescriptions by right. One physician said:

"We either swing the knife or use the Dictaphone." (Senior house officer (5))

\section{Conflicts with existing routines Low priority task}

The physicians on call often had to prioritise urgent clinical assignments. They possessed diverse views on nurses' requests to clear up ambiguous drug prescriptions: some regarded these requests as unnecessary and annoying interruptions while others considered them as convenient precautions. One nurse described how she encountered this in daily clinical practice:

"Some of the physicians might as well be looking out the window when they sign for the prescriptions." (Charge nurse (7))

Some of the participants felt that physicians generally gave priority to research and downgraded clinical work. Several physicians argued that the patients would benefit more if physicians spent time on professional specialisation instead of completing drug prescribing sheets:
"What is the big idea of writing dates on drug sheets? Isn't it more important to improve your skills in ECG?" (Senior house officer (3))

Some of the nurses considered drug administration as dull routine work while others regarded it as very important. One male nurse felt that at times nurses were prompted to exceed their powers by administering medications which the physicians had not signed for:

"These situations will emerge on acute wards. It must not become an obstacle to the work with the other patients. You must get along." (Nurse (8))

The participants said that the patient was the theoretical pivotal point for all staff activity. They agreed that errors of drug treatment were undesirable but there were diverse views on pursuing this aim by introducing new drug prescribing procedures.

\section{Logistical problems}

In a few situations drug prescribing sheets were not accessible to the physicians-for example, patients being sent to the outpatient clinic without their drug chart. The consultants therefore had to make assumptions about the patients' medications and to give conditional advice. On each ward section nursing staff stored the drug chart folder in different places and most physicians had had a problem in finding the drug prescribing sheets. When sent for, they had spent minutes looking for the folders. To some physicians these were minor problems while, to others, these logistical problems contributed to the general view of a malfunctioning drug prescribing system.

\section{DISCUSSION}

This study has shown that the use of drug prescribing sheets on two medical wards met with resistance. Nine different problems were identified at the individual, group, and organisational level 8-16 months after the sheets were first used. Incompetence in using the drug prescribing sheet created resistance and forced physicians and nurses to make their own interpretations and to create solutions to the problems they faced. In addition, the new procedures conflicted with elements of existing structure, culture, and routines.

Within the new system nurses have taken on the responsibility for storing the drug chart folders. Secretaries still organise the progress notes but they no longer interpret and type badly recorded drug prescriptions, which might explain why problems like poor handwriting, unclear prescribing, and logistical problems seem to be more of a problem with the new system.

The drug prescribing sheet is not to be regarded only as a tool but, in a broader sense, as an endogenous organisational entity created and controlled through the interaction of staff, process, and artefact. Freedom to order and change medications, freedom to adopt new treatment procedures, and freedom to set volume of work and scheduled priorities have been related to physicians' perceived clinical autonomy and physician satisfaction. ${ }^{20}{ }^{21}$ By forcing staff to modify procedures of prescribing, adoption of the drug prescribing sheets may have generated general dissatisfaction and created resistance.

Conflicts between physicians and nurses about drug prescribing sheets have been reported previously. ${ }^{32}$ The nurses and physicians appeared to have different positions within the department organisation, representing two different subcultures. Organisational culture includes established routines, norms, and prevailing attitudes and values. ${ }^{33}$ A study on the professional cultures in Danish hospital departments has previously revealed differences between physicians and nurses with the potential for conflict. ${ }^{34}$ Although it was beyond the scope of the present study to perform a complete cultural analysis, the findings support this study of physician and 
nurse cultures. The explanation for many of the problems identified includes the possibility that physicians and nurses differ in their acceptance of routine tasks. The nurses were characterised by collectivism while the physicians were more competitive and individualistic and orientated towards technology and science. A surprising finding was the contrast between these values and the espoused value ${ }^{33}$ : the patient is the pivotal point for our activities. The managerial challenge is to promote collaboration by balancing these cultural differences.

This study shows that lack of awareness of procedures, insufficient dissemination of knowledge, and collegial difficulty and scepticism among those who put drug handling into practice is likely to have a prolonged impact on service quality. According to Mintzberg the structure of hospital wards is characterised by low flexibility and marked professional autonomy. ${ }^{35}$ Temporary resistance to change is to be expected ${ }^{36}$ but the experiences and beliefs of health professionals may cause strong and more persistent resistance to occur. ${ }^{37}$ Thus, change in professional behaviour seems to depend on cultural changes ${ }^{37-41}$ including changes in attitude towards improving skills. ${ }^{35}$ The solutions to the identified problems may be divided into two distinct but overlapping groups: (1) issues for ward managers such as providing sufficient training, establishing clear roles and responsibilities, and solving logistical problems, and (2) involvement of staff in developing teamwork and providing an understanding of the comprehensive drug process including sources of error. Meeting the expectation of involvement among staff appears to be crucial, but the departments must be in control of the process and maintain the momentum for change throughout the entire process. One of the challenges is to reward behaviour that supports the change and to weaken the restraining forces.

The choice of in depth interviews rather than a cross sectional survey may be justified by the detailed data collected. Interviews are more appropriate than quantitative methods when the aim is to study the complex phenomena of organisational and interpersonal issues. ${ }^{42}{ }^{43}$ Information which cannot be reached by other research methods on concealed and taboo subjects within an organisation may be unveiled by interview. ${ }^{44}$ The method is flexible and allows the investigator to follow up the individual way in which respondents interpret and answer. Categories are developed to reflect as many of the nuances in the data as possible. By an iterative comparison of each item with the rest of the data, complexity can be embraced. These benefits need to be balanced against the limitations associated with the small number of participants. The method produces an increased understanding of a small number of people but reduces external validity and generalisability. ${ }^{25}$ To increase study validity participants were recruited from two different settings by a guided sampling approach. Throughout the entire study steps were taken to increase validity, including the use of a confirmatory interview technique and an explicit analysis approach, informant confirmation, and triangulation. However, the disadvantage of a single researcher conducting the interviews and analysing the data still needs to be considered. ${ }^{3144}$

The aim of this study was to identify difficulties faced by physicians and nurses as they use drug prescribing sheets. Although the problems identified were related to a specific task, they may be obstacles of a more universal nature-for example, similar problems have recently been reported as obstacles to the postgraduate training of Danish physicians, including problems related to the organisation of work, non-systematic and insufficient introduction of new staff, low group cohesiveness, and low exchange of experiences. ${ }^{45}$ To overcome resistance and provide quality development these obstacles must be taken into account and solved. This study suggests that more profound cultural and structural problems are crucial to the implementation of the new drug record system. However, only a few of the identified problems appear to

\section{Key messages}

- There is an increasing awareness and concern over medication errors.

- Implementation of a single drug prescribing sheet, used by physicians to write all medication orders and by nurses during dispensing and administration of medications, may improve the quality of health care primarily by reducing the number of drug prescribing errors.

- Physicians and nurses differed in their degree of acceptance of the new system, and difficulties relating to culture and structure were crucial to its implementation.

- More fundamental interventions than training in the use of the new system are required to achieve the intended changes in the procedures of drug prescription and administration.

- Adoption of drug prescribing sheets forces staff to develop interdisciplinary collaboration.

have a straightforward solution. For example, problems regarding job identity should, to some extent, be solved outside the clinical setting because cultural values are gradually acquired during the course of medical training. ${ }^{46} \mathrm{~A}$ stepwise approach, combining interventions that focus on social interaction and organisational adaptation, appears more appropriate to improve the quality of systems by which drugs are ordered and administered than training alone. As a result of the present study we have changed our implementation strategy for the new system which now includes specific training, enhanced leadership, use of local opinion leaders, formation of local networks, and iterative audit and feedback.

\section{ACKNOWLEDGEMENTS}

This study was conducted while the author was a research fellow in clinical pharmacology in Bispebjerg Hospital. The author thanks the Danish Institute for Health Technology Assessment and Copenhagen Hospital Cooperation for their financial support.

\section{Author's affiliation}

S E Andersen, H:S Bispebjerg Hospital, Section of Clinical Pharmacology, Copenhagen and H:S Amager Hospital, Centre of Internal Medicine, Copenhagen, Denmark

\section{REFERENCES}

1 Trillwood W. Administration of drugs in hospitals (letter). Lancet 1959;355.

2 Senderovitz T, Christophersen AB, Christensen HR, et al. Discrepancies between medical records and dispensing records in two large hospital departments in Copenhagen (in Danish). Ugeskr Laeger 1998; 160:4055-8.

3 Watt JA, Dorricott N, Stewart RA, et al. Methods of recording prescriptions in hospital and their effects on transcribed drug lists. $\mathrm{Br} J$ Psychiatry 1973;1 22:65-70.

4 Siple JF, Joseph CL. Medication discrepancies on admission to a nursing home. Am J Hosp Pharm 1992;49:397-400.

5 Andersen SE, Fog D. Written documentation of drug prescriptions. Accordance between medical records and dispensing records (in Danish). Ugeskr Laeger 1998;160:4059-62.

6 Leape LL, Bates DW, Cullen DJ, et al. Systems analysis of adverse drug events. ADE Prevention Study Group. JAMA 1995;274:35-43.

7 Weingart SN, Wilson RM, Gibberd RW, et al. Epidemiology of medical error. BM 2000:320:774-7.

8 Leape LL. Error in medicine. JAMA 1994;272:1851-7.

9 Bates DW. Medication errors. How common are they and what can be done to prevent them? Drug Safety 1996;15:303-10.

10 Bates DW, Cullen DJ, Laird N, et al. Incidence of adverse drug events and potential adverse drug events. Implications for prevention. ADE Prevention Study Group. JAMA 1995;274:29-34.

11 Crooks J, Clark CG, Caie HB, et al. Prescribing and administration of drugs in hospital. Lancet 1965;ii:373-8.

12 Crooks J, Weir RD, Coull DC, et al. Evaluation of a method of prescribing drugs in hospital, and a new method of recording their administration. Lancet 1967;i:668-71.

13 Bergman U, Norlin A, Wiholm BE. Inadequacies in hospital drug handling. Acta Med Scand 1979;205:79-85.

14 Håberg M. Evaluation of the old and new system for ordinating medicines at Sentralsjukehuset i Rogaland autumn 1990/spring 1991 and 1992 (in Norwegian). Tidskr Nor Laegeforen 1992;11 12:2378-82. 
15 Wyatt JC, Wright P. Design should help use of patients' data. Lancet 1998:352:1375-8.

16 Hill PA, Wigmore HM. Measurement and control of drug-administration incidents. Lancet 1967;i:671-4.

17 Gardulf A, Nordström G. Correctly written drug prescriptions important for the nurses' drug handling. Vård i Norden 1998;18:4-8.

18 Schousboe LP, Tandrup O. Drug prescription documentation (in Danish). Ugeskr Laeger 1999;161:4389-92.

19 Oxman AD, Thomson MA, Davis DA, et al. No magic bullets: a systematic review of 102 trials of interventions to improve professional practice. Can Med Assoc J 1995;153:1423-31

20 Schulz R, Schulz C. Management practices, physician autonomy, and satisfaction. Evidence from mental health institutions in the Federal Republic of Germany. Med Care 1988;26:750-63.

21 Barr DA. The effects of organizational structure on primary care outcomes under managed care. Ann Intern Med 1995;122:353-9.

22 Changing professional practice. DSI Report 99.05. Copenhagen: DSI Danish Institute for Health Services Research and Development, 1999.

23 Grol R. Beliefs and evidence in changing clinical practice. BM 1997;315:418-21.

24 Robertson N, Baker R, Hearnshaw $\mathrm{H}$. Changing the clinical behavior of doctors: a psychological framework. Qual Health Care 1996:5:51-4.

25 Patton MQ. Qualitative evaluation and research methods. Newbury Park, California: Sage Publications Inc, 1990

26 Leavit HJ. Organizational change in industry: structural, tecnological and humanistic approaches. In: March JG, ed. Handbook of organizations. Chicago: Rand McNally \& Company, 1965.

27 Kvale S. Interview. En introduktion til det kvalitative forskningsinterview. Copenhagen: Hans Reizels Forlag, 1994.

28 Malterud K. Shared understanding of the qualitative research process. Guidelines for the medical researcher. Fam Pract 1993;10:201-6.

29 Malterud K. Qualitative methods in medical research. An introduction (in Norwegian). Oslo: Tano Aschehoug, 1996.

30 Weitzman EA. Analyzing qualitative data with computer software. Health Serv Res 1999;34:1241-63.

31 Patton MQ. Enhancing the quality and credibility of qualitative analysis. Health Serv Res 1999; 34:1189-208.
32 Sanner J. A conflict between physicians and nurses about the drug lists. Please, do not disturb - the drug documentation is going on (in Danish). Lakartidningen 1998;95:2728-32.

33 Schein EH. Organisational culture and leadership. Holte: Forlaget Valmuen, 2000

34 Eriksen $\mathbf{H}$, Ulrichsen $\mathrm{H}$. Three cultures in the hospital sector (in Danish). Copenhagen: Handelshøjskolens forlag, 1991.

35 Sorensen NB. Forms and cultures of organisations. About Mintzberg's theory in a Danish context (in Danish). Copenhagen: Samfundslitteratur, 2000.

36 Garside P. Organisational context for quality: lessons from the fields of organisational development and change management. Qual Health Care 1998;7(Suppl):S8-15.

37 Haines A, Donald A. Making better use of research findings. BM 1998;317:72-5.

38 Davies HT, Nutley SM, Mannion R. Organisational culture and quality of health care. Qual Health Care 2000:9:111-9.

39 Koeck C. Time for organisational development in healthcare organisations. Improving quality for patients means changing the organisation (editorial). BM 1998;317:1267-8.

40 Donaldson LJ, Gray JA. Clinical governance: a quality duty for health organisations. Qual Health Care 1998;7(Suppl):S37-44.

41 Davies HT, Nutley SM. Developing learning organisations in the new NHS. BM 2000;320:998-1001.

42 Pope C, Mays N. Qualitative research: reaching the parts other methods cannot reach: an introduction to qualitative methods in health and health services research. BM 1995;311:42-5.

43 Fitzpatrick R, Boulton M. Qualitative methods for assessing health care. Qual Health Care 1994;3:107-13.

44 Borum F. The analysis of organisations by qualitative interview methods (in Danish). Copenhagen: Handelshøjskolen i København, 1998.

45 Utzon J. Hospital physicians: organisation of work, education, satisfaction, and productivity (in Danish). DSI Report 98.02. Copenhagen: DSI Danish Institute for Health Services Research and Development, 1998

46 Borum F. Inertia and change options in the hospital field (in Danish). In: Hildebrandt S, Schultz $M$, eds. Hospital management. Copenhagen: Munksgaard, 1997: 263-82.

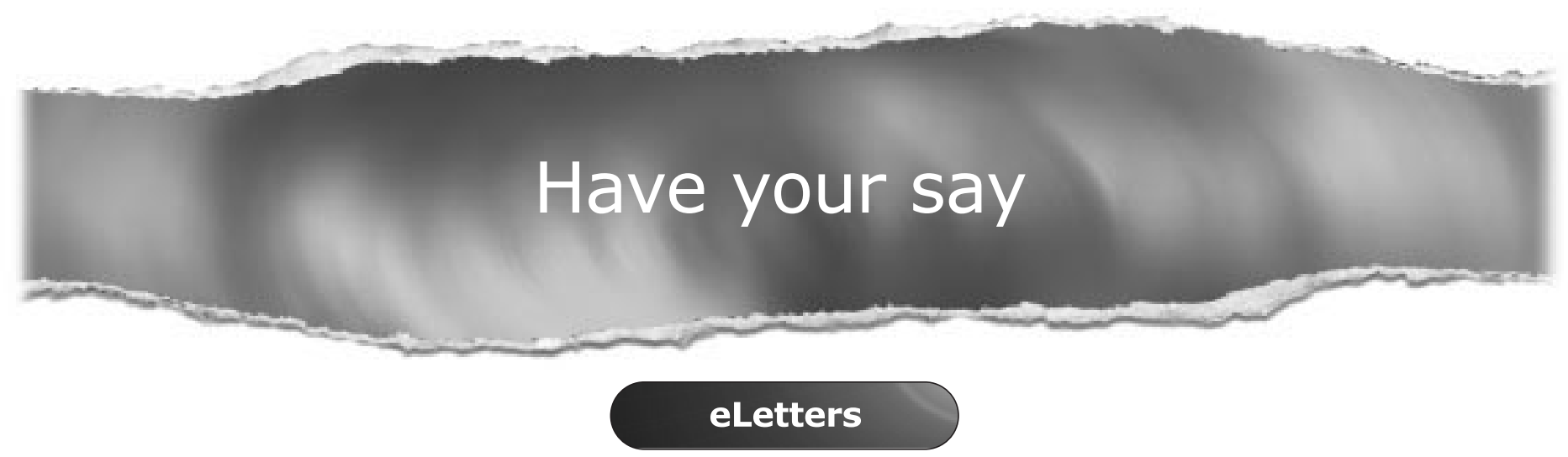

If you wish to comment on any article published in Quality in Health Care you can send an eLetter using the eLetters link at the beginning of each article. Your response will be posted on Quality in Health Care online within a few days of receipt (subject to editorial screening).

\section{www.qualityhealthcare.com}

